\title{
Posterior Malleolus Fractures in Trimalleolar Fracture Dislocations of the Ankle Fixed with Anteroposterior Screws vs Posterior Plating: A Clinical Outcome Study
}

\author{
Arunkamal Chandramohan ${ }^{1}$, Dheenadhayalan Jayaramaraju ${ }^{2}$, Devendra Agraharam ${ }^{3}$, Ramesh Perumal ${ }^{4}$, \\ Rajasekaran Shanmuganathan ${ }^{5}$
}

\begin{abstract}
Introduction: Fracture dislocations of the ankle are rare injuries with often poor outcomes due to posttraumatic arthritis and instability of the ankle. A concomitant fracture of the posterior malleolus occurs in $7-44 \%$ of all ankle fractures.

Aim of the study: To study the outcomes in operated cases of posterior malleolus fractures fixed using percutaneous anteroposterior (AP) screws vs posterior buttress plating and to study which is advantageous.

Materials and methods: Our study was conducted between December 2013 and December 2015. All operated cases of posterior malleolus fractures in trimalleolar fracture dislocations of the ankle joint were included. Polytrauma cases and open injuries were excluded. Among 28 cases, 24 cases were available for final follow-up. The average age was 47.2 years, and road traffic accident was the predominant cause in $80 \%$ of the cases. In 9 cases, buttress plating, and in 15 cases, percutaneous AP screws were used as decided by senior surgeon. All cases were followed up at periodic intervals. Mean follow-up is 28.5 months.

Results: Average union time is 14.2 weeks. Ankle fracture scoring system designed by Phillips et al. was used. The outcome is the sum of the clinical score (follow-up), the anatomical score (postoperative), and arthritis score (follow-up). A minimum score is 0 , and the maximum score is 150 . In posterior plating group, the average score was 128 out of 150. In AP screws group, the average score was 114 out of 150 . Improper reduction and articular step were noticed in three cases and developed ankle arthritis in subsequent radiographs. There were no infections in our series. Conclusion: Posterior plating gave better results due to stable anatomical reduction and by early physiotherapy, while AP screws group had relatively moderate outcome due to articular step off and late arthritic changes.

Keywords: Fracture dislocation ankle, Percutaneous AP screws, Posterior buttress plate, Posterior malleolus fracture, Surgical outcome.

Journal of Orthopedics and Joint Surgery (2019): 10.5005/jp-journals-10079-1008
\end{abstract}

\section{INTRODUCTION}

Fracture dislocations of the ankle are rare injuries with often poor outcomes due to posttraumatic arthritis and instability of the ankle. A concomitant fracture of the posterior malleolus occurs in $7-44 \%$ of all ankle fractures. ${ }^{1,2}$ The size of the fragment, usually given as the percentage of involvement of the distal tibial articular surface as measured on the lateral ankle radiograph, is the most important parameter used to decide whether a fragment should be fixed. A higher incidence of posttraumatic osteoarthritis (OA) and posterior instability of the talus with larger fragments has led to the generally accepted algorithm that fragments larger than $25 \%$ of the articular surface should be fixed. ${ }^{3,4}$ However, in fracture dislocations of the ankle, all posterior malleolar fractures should be fixed to retain the stability of ankle mortise.

\section{Aim of the Study}

To study the outcomes in operated cases of posterior malleolus fractures in trimalleolar fracture dislocations of the ankle, fixed using AP cancellous screws vs posterior buttress plating and to study which is advantageous.

\section{Materials and Methods}

Our study was conducted between December 2013 and December 2015. Our study was a nonrandomized study using consecutive case series. All operated cases of posterior malleolus fractures in
${ }^{1}$ Department of Orthopaedics, Ganga Medical Centre and Hospitals Private Limited, Coimbatore, Tamil Nadu, India

${ }^{2-4}$ Department of Orthopedics and Trauma, Ganga Medical Centre and Hospitals Private Limited, Coimbatore, Tamil Nadu, India

${ }^{5}$ Department of Orthopaedics and Spine Surgery, Ganga Medical Centre and Hospitals Private Limited, Coimbatore, Tamil Nadu, India

Corresponding Author: Arunkamal Chandramohan, Department of Orthopaedics, Ganga Medical Centre and Hospitals Private Limited, Coimbatore, Tamil Nadu, India, Phone: +91 9751405855, e-mail: arunkamalch@gmail.com

How to cite this article: Arunkamal C, Dheenadhayalan J, Agraharam D, et al. Posterior Malleolus Fractures in Trimalleolar Fracture Dislocations of the Ankle Fixed with Anteroposterior Screws vs Posterior Plating: A Clinical Outcome Study. J Orth Joint Surg 2019;1(1):11-14.

Source of support: Nil

Conflict of interest: None

trimalleolar fracture dislocations of the ankle joint were included. Only trimalleolar fractures without ankle dislocations were excluded. Polytrauma cases and open injuries of the ankle were excluded.

Among 28 cases, 24 cases were available for final follow-up at the end of 2 years. There were 12 males and 12 females. 
The road traffic accident was the predominant cause in $80 \%$ of cases, followed by fall from height. The posterior malleolus buttress plating and fibula plating were done through posterolateral approach in 9 cases, and in 15 cases, percutaneous AP $4 \mathrm{~mm}$ cancellous screws fixation for posterior malleolar fracture was used. The choice of implant was decided by senior operating surgeon after studying the radiographs of the ankle. For those patients with single large posterior malleolar fracture fragment, AP screws were used by percutaneous technique, and whenever more than one posterior malleolar fragment was present or associated with comminution, posterior buttress plate fixation technique was chosen.

\section{Surgical Technique-posterolateral Approach for Plating}

A longitudinal incision was made between the lateral border of the achilles tendon and the medial border of the fibula. Fixation of the fibula was attempted first in most cases, and that led to an indirect reduction in the posterior malleolar fracture due to attachment of posterior tibiofibular ligament. Care was taken to avoid injury to the sural nerve. The posterior aspect of the fibula was reached through the interval just lateral to the peroneal tendon. By retracting the muscle belly flexor hallucis longus medially, the posterior aspect of the tibia can be seen. Through the interval medial to the peroneal tendon, particular care was taken to avoid injury to the peroneal artery and its smaller branches. Posterior 3.5/4.5 stainless steel buttress plates were used to reduce and to hold the posterior malleolar fracture fragment under image intensifier guidance (Fig. 1).

\section{Percutaneous AP Screws Technique}

Using gentle traction, ankle joint dislocation was reduced. First, the fibula fracture was fixed using conventional lateral approach with one-thired of tubular plate and screws and then by maximal dorsiflection of the ankle, and one or two $\mathrm{k}$ wires were passed percutaneously from posterior aspect into posterior malleolar fracture and can be used as a joystick to reduce the posterior fragment and then secured temporarily with $\mathrm{K}$ wires anteriorly. Indirect reduction can be achieved using Weber clamp. A near anatomical reduction was almost always achieved, and fracture fragments were held with $4 \mathrm{~mm}$ cancellous screws in the anteroposterior (AP) direction. Finally, medial malleolus fracture was fixed with tension band wiring technique or with cancellous screws (Fig. 2).

All cases were followed up at periodic intervals at the time of suture removal, 4, 8, 12, 16, 24 weeks and thereafter once in 6 months until final follow-up. Mean follow-up period is 28.5 months (range 15-34 months). Average patient's age is 47.2 years (range from 28 years to 75 years). In all cases, closed reduction was done within 8 hours since the injury, and in seven cases with tense swelling, ankle spanning external fixator was done and later converted to definitive internal fixation. The average time delay from injury to definitive surgery was 5.3 days (range 3 days to 14 days) (Tables 1 and 2).

\section{Results}

Average union time is 14.2 weeks in plating group and 15.5 weeks in AP screws group. Ankle fracture scoring system designed by
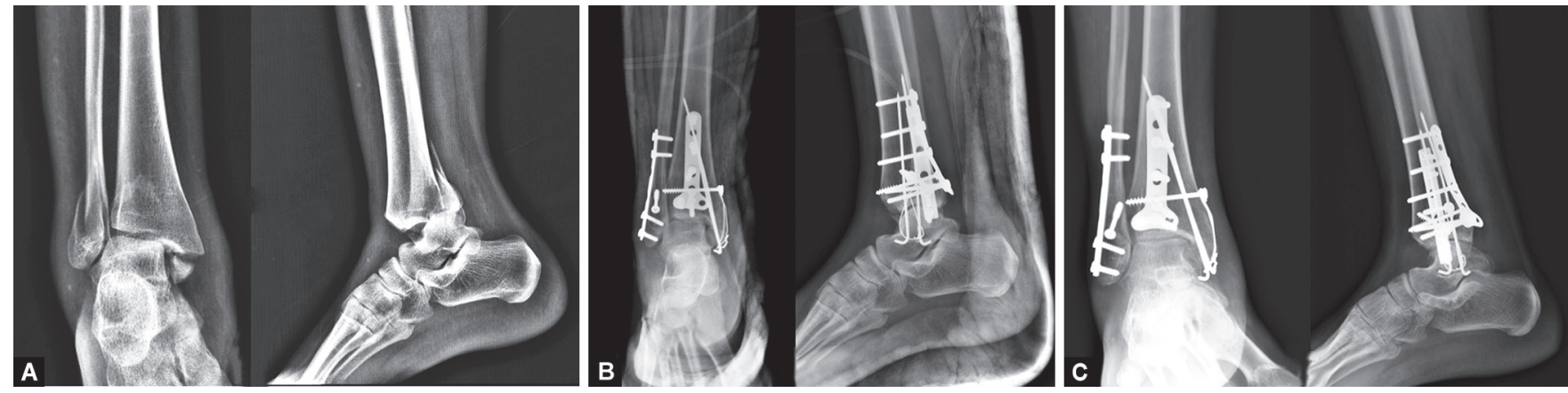

Figs $1 \mathrm{~A}$ to C: Posterior plating done for posterior malleolus fracture in a female patient, aged 55 years, fell from ladder: (A) Preoperative $\mathrm{X}$ rays; (B) Immediate postoperative X-rays; (C) Final postoperative X-rays
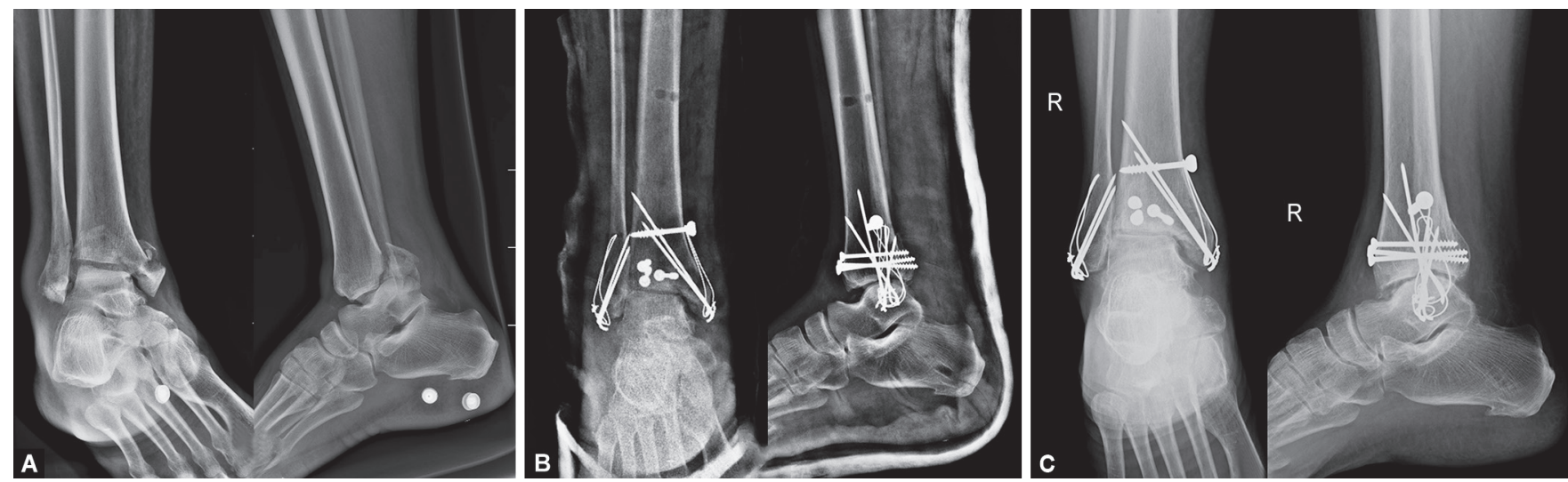

Figs 2 A to C: Posterior malleolus fixed with AP screws in a male patient, aged 57 years, fell from two wheeler: (A) Preoperative X rays; (B) Postoperative $X$-rays at the end of second month—articular step with posterior joint space narrowing; (C) After 2 years—secondary ankle arthritis 
Table 1: Case distribution

\begin{tabular}{ll}
\hline Total patients & 24 cases \\
Age in years & $(47.2)$ mean \\
Males : females & $12: 12$ \\
RTA : fall from height & $16: 8$ \\
Pronation injuries & 14 cases \\
Supination injuries & 10 cases \\
AP screws & 15 cases \\
Posterior plating & 9 cases \\
\hline
\end{tabular}

Table 2: Clinical outcome

\begin{tabular}{lll}
\hline Results & AP screws & Posterior plate \\
\hline Union time & 15.5 weeks & 14.2 weeks \\
Anatomical reduction & $12 / 15$ cases & $9 / 9$ cases \\
Articular step & 3 cases & No step \\
Painful arthritis & 2 cases & No cases \\
Good to excellent results & $12 / 15$ cases & $8 / 9$ cases \\
Implant failure & None & None \\
Phillips ankle score (out of 150$)$ & 114 & 128 \\
\hline
\end{tabular}

Phillips et al. ${ }^{5}$ was used. The outcome is the sum of the clinical score (follow-up), the anatomical score (postoperative), and arthritis score (follow-up). A minimum score is 0 , and the maximum score is 150 . In posterior plating group, the average score was 128 out of 150 .

In AP screw group, average ankle hind foot score was 114 out of 150. Two screws were used in 5 cases, and 1 screw in 10 cases. There were no infections or implant loosening in our series. In AP screws group, two patients had grade III arthritic changes in the ankle joint with restricted dorsiflection and pain but were treated with analgesics and footwear modification.

\section{Discussion}

In a systematic review article ${ }^{6}$ published in the Journal of Foot \& Ankle Surgery (2015), the authors concluded that based upon the clinical and biomechanical studies, they identified the following three factors that will prognosticate ankle injuries outcome, namely, the presence of fracture-dislocation at the injury, articular surface congruity, and residual talar subluxation.

The results of the biomechanical studies confirmed that posterior ankle stability is predominantly provided by anterior inferior tibiofibular ligament, posterior inferior tibiofibular ligament, and medial and lateral structures with little contribution from posterior malleolus. Although in itself the posterior malleolus might not be involved in significant load bearing at the tibiotalar joint, with posterior malleolus fractures, the redistribution of the load is abnormal, which might predispose the patient to the development of posttraumatic arthritis. ${ }^{7}$ Patients with a persistent articular step of $>1 \mathrm{~mm}$ after fixation of the posterior malleolus were significantly more likely to develop posttraumatic OA than those with no articular step ( $46 \%$ vs $25 \%, p=0.02)$. ${ }^{2,8}$

Usually, the posterior malleolus is only fixed if the fragment exceeds $25 \%$ of the articular surface or if there is an instability of the ankle joint after fixation of the medial and lateral malleoli. However according to the studies in orthopedic literature, whenever a posterior malleolus fracture is associated with fracture dislocation of the ankle, the surgeon should have a low threshold for surgical fixation. This should be confirmed intraoperatively after assessing residual talar subluxation and joint congruity. ${ }^{9}$
In the percutaneous reduction with AP screw fixation of the posterior malleolus, anatomical reduction can be difficult due to the interposition of soft tissue or loose bony fragments; it is hard to assess reduction satisfactorily using an image intensifier, and the fixation of small or comminuted fragments is technically difficult. Incomplete reduction leads to a residual step in the articular surface. ${ }^{8,10}$ However, in posterior buttress plating group, anatomical reduction was done under vision through posterolateral approach, and fibula fracture was also fixed through same approach. Only medial malleolus requires separate skin incision and fixation by cancellous screws or tension band wires.

In a biomechanical study ${ }^{11}$ of buttress posterior plating vs AP lag screws for fixation of the posterior malleolus fractures, posterior malleolus fractures involving $30 \%$ of the distal tibial articular surface were created in seven pairs of fresh frozen cadaveric ankles. One specimen in each pair was randomly assigned to fixation with either two AP lag screws or a buttress plate without supplemental lag screws. Each specimen was then subjected to cyclic loading from $0 \%$ to $50 \%$ of body weight for 5,000 cycles followed by loading to failure. Outcome measures included permanent axial displacement during each test cycle. They concluded that posterior malleolus fractures treated with posterior buttress plating showed significantly less displacement during cyclical loading compared with fractures fixed with AP lag screws.

Haraguchi et al. ${ }^{12}$ classified posterior malleolus fractures using computed tomography into three types: (1) posterolateral oblique type (67\%), (2) medial extension type (19\%), and (3) small shell type (14\%). This article emphasizes the importance of CT scans of the involved ankle in the initial assessment and preoperative planning of fracture dislocations of the ankle.

\section{Conclusion}

The posterior plate had a better radiological and functional outcome due to stable anatomical reduction through posterolateral approach. The AP screw group had nonanatomical reduction of the articular fragments due to soft tissue interposition and subsequent arthritic changes in our series. For large single posterior malleolus fracture, two AP $4 \mathrm{~mm}$ cancellous screws can be used using percutaneous Weber clamp, after confirming reduction using image intensifier. For comminuted posterior malleolus fracture, posterior buttress plate is really advantageous. In the future, for a better understanding of these fractures, CT scan-based classification of posterior malleolar fractures would be of paramount importance.

\section{References}

1. Van den Bekerom MP, Haverkamp D, Kloen P. Biomechanical and clinical evaluation of posterior malleolar fractures. A systematic review of the literature. J Trauma 2009;66(1):279-284. DOI: 10.1097/ TA.0b013e318187eb16.

2. McDaniel WJ, Wilson FC. Trimalleolar fractures of the ankle. an end-result study. Clin Orthop Relat Res 1977(122):37-45. DOI: 10.1097/00003086-197701000-00006.

3. Drijfhout van Hooff CC, Verhage SM, Hoogendoorn JM. Influence of fragment size and postoperative joint congruency on longterm outcome of posterior malleolar fractures. Foot Ankle Int 2015;36(6):673-678. DOI: 10.1177/1071100715570895.

4. Xu HL, Li X, Zhang DY, et al. A retrospective study of posterior malleolus fractures. Int Orthop 2012;36(9):1929-1936. DOI: 10.1007/ s00264-012-1591-9.

5. Phillips WA, Schwartz HS, Keller CS, et al. A prospective, randomized study of the management of severe ankle fractures. J Bone Joint Surg Am 1985;67(1):67-78. DOI: 10.2106/00004623-198567010-00010. 
6. Odak S, Ahluwalia R, Unnikrishnan P, et al. Management of posterior malleolar fractures: a systematic review. J Foot Ankle Surg 2016;55(1):140-145. DOI: 10.1053/j.jfas.2015.04.001.

7. Langenhuijsen JF, Heetveld MJ, Ultee JM, et al. Results of ankle fractures with involvement of the posterior tibial margin. J Trauma 2002;53(1):55-60. DOI: 10.1097/00005373-200207000-00012.

8. Mingo-Robinet J, López-Durán L, Galeote JE, et al. Ankle fractures with posterior malleolar fragment: management and results. J Foot Ankle Surg 2011;50(2):141-145. DOI: 10.1053/j.jas.2010.12.013.

9. de Vries JS, Wijgman AJ, Sierevelt IN, et al. Long-term results of ankle fractures with a posterior malleolar fragment. J Foot Ankle Surg 2005;44(3):211-217. DOI: 10.1053/j.jfas.2005.02.002.
10. Gardner MJ, Streubel PN, McCormick JJ, et al. Surgeon practices regarding the operative treatment of posterior malleolus fractures. Foot Ankle Int 2011;32(4):385-393. DOI: 10.3113/FAI.2011. 0385.

11. Bennett $C$, Behn A, Daoud A, et al. Buttress plating versus anteriorto-posterior lag screws for fixation of the posterior malleolus: a biomechanical study. J Orthop Trauma 2016;30(12):664-669. DOI: 10.1097/BOT.0000000000000699.

12. Haraguchi N, Haruyama $\mathrm{H}$, Toga $\mathrm{H}$, et al. Pathoanatomy of the posterior malleolar fracture of the ankle. J Bone Joint Surg Am 2006;88(5):1085-1092. DOI: 10.2106/00004623-20060500000020 . 